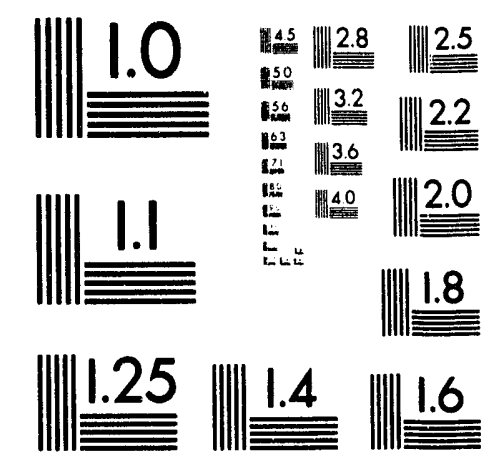



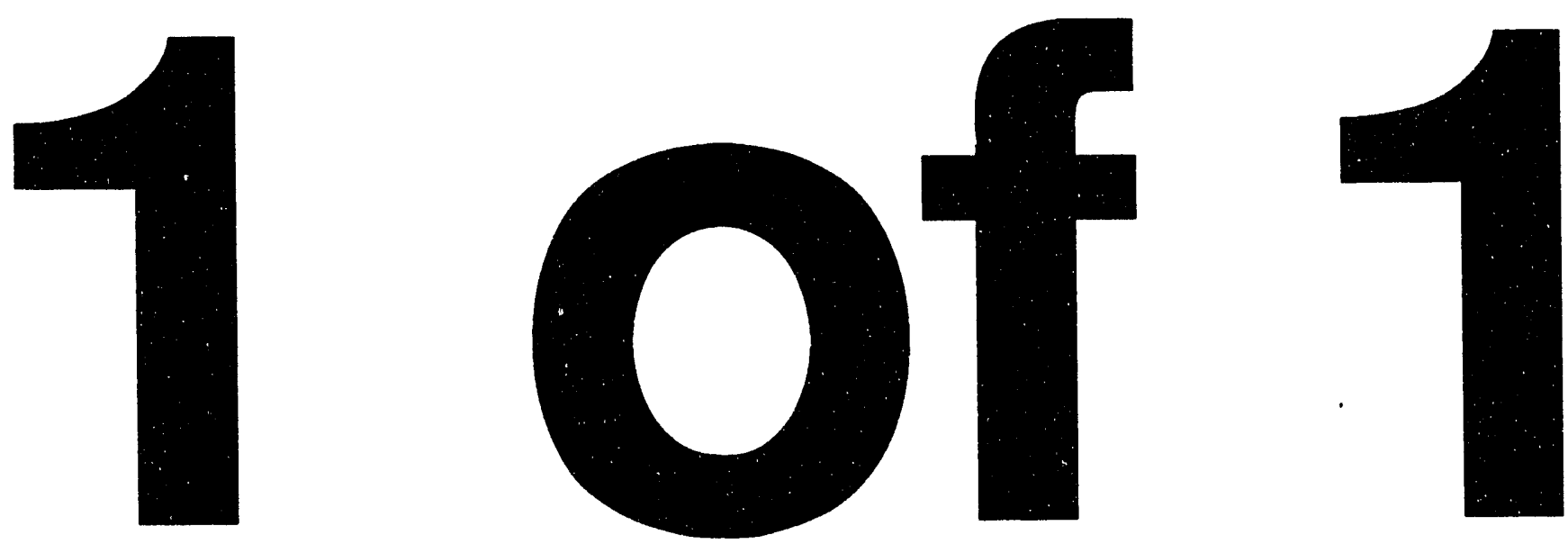
L.BL-33240

UC-410

LSGN-139

\title{
COMMISSIONING AND PERFORMANCE OF THE ADVANCED LIGHT SOURCE
}

\author{
ALAN JACKSON \\ ACCELERATOR and FUSION RESEARCH DIVISION \\ Lawrence Berkeley Laboratory \\ University of California \\ Berkeley, CA 94720
}

MAY 1993

This work was supported by the Director, Office of Energy Research, Office of Basic Energy Sciences, Materials Sciences Division, of the U.S. Department of Energy under Contract No. DE-AC03-76SF00098. 


\title{
Commissioning and Performance of the Advanced Light Source
}

\author{
Alan Jackson \\ Lawrence Berkeley Laboratory, University of California, Berkeley, California 94720
}

\section{Abstract}

The Advanced Light Source (ALS) at the Lawrence Berkeley Laboratory is the first of the lower eneigy (1- to 2$\mathrm{GeV}$ ) third-generation synchrotron radiation facilities to come into operation. Designed with very small electron beam emittances, to operate with long insertion devices to produce very high brightness beams of synchrotron radiation in the VUV and soft $x$-ray regions of the spectrum, these facilities are complementary to the higher energy (6- to $9-\mathrm{GeV}$ ) facilities 1 designed for harder $\mathrm{x}$-radiation. From the earliest periods of their design it was recognized that the performance of the required low-emittance lattices would be dominated by nonlinear particle dynamics catised $b_{j}$ the strong lattice sextupoles required for chromatic correction, the effects of undulators and wigglers, and the requirement for very narrow gap vacuum vessels. Commissioning of the ALS storage ring began early in 1993. In this paper we will briefly review the main characteristics of the storage ring design, describe our commissioning experiences, and review the present performance and performance limitations of the facility.

\section{INTRODUCTION}

The Advanced Light Source at the Lawrence Berkeley Laboratory is the first of the lower energy (1- to 2-GeV) thirdgeneration synchrotron radiation facilities to come into operation. Designed with very small electron beam emittances, to operate with long insertion devices to produce very high brightness beams of synchrotron radiation in the VUV and soft $x$-ray regions of the spectrum, these facilities are complementary to the higher energy (6- to 9-GeV) facilities designed for harder $\mathrm{x}$-radiation. From the earliest periods of their design it was recognized that the performance of the required low-emittance lattices, which utilize relatively strong focusing quadrupoles, would be dominated by nonlinear particle dynamics caused by the powerful lattice sextupoles required for chromatic correction. The dynamics are also modified by the fields of the undulator and wiggler magnets (collectively known as insertion devices, or IDs), and their requirement for very narrow-gap vacuum vessels. However, neither the IDs, nor their narrow-gap chambers, were installed during the commissioning period reported here.

The ALS storage ring commissioning was divided into two phases: January 13 to March 15 1993, when beam could be injected but not accelerated, and March 16 on, after the RF system was brought into operation. Commissioning activities were curtailed on April 30 in order to install two IDs and their beamlines, three bend-magnet beamlines, and a diagnostic beamline. Other shutdown activities include an in-situ bake and realignment of half of the storage ring girders.
In this paper we will briefly review the main characteristics of the storage ring design, outline the chronology of the commissioning period, review the performance and performance limitations of the facility, and describe our commissioning experiences, concentrating on the second phase when RF was available.

\section{LATTICE DESIGN}

The ALS storage ring lattice is based on a triple-bend achromat configuration with twelve-fold symmetry. A compact structure is achieved by including vertical focusing gradients in the bend magnets, using a single quadrupole family (QFA) to control the dispersion function, a quadrupole doublet family (QF and QD) to match the betatron tunes, and two families of sextupoles (SF and SD) to correct the chromaticity, in the arrangement shown in Figure 1. Small natural emittance ( $3.5 \mathrm{~nm}$ at the nominal energy of $1.5 \mathrm{GeV}$ ) is ensured through the juxtaposition of low values of dispersion and horizontal beta-function (and their derivatives) in the bend magnets. The major lattice parameters are shown in Table 1.

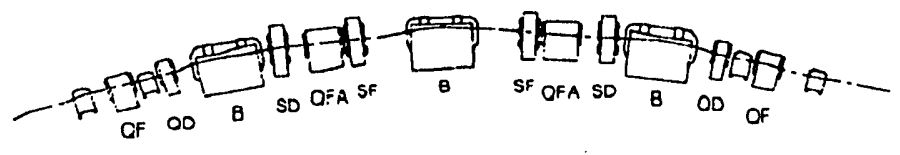

a

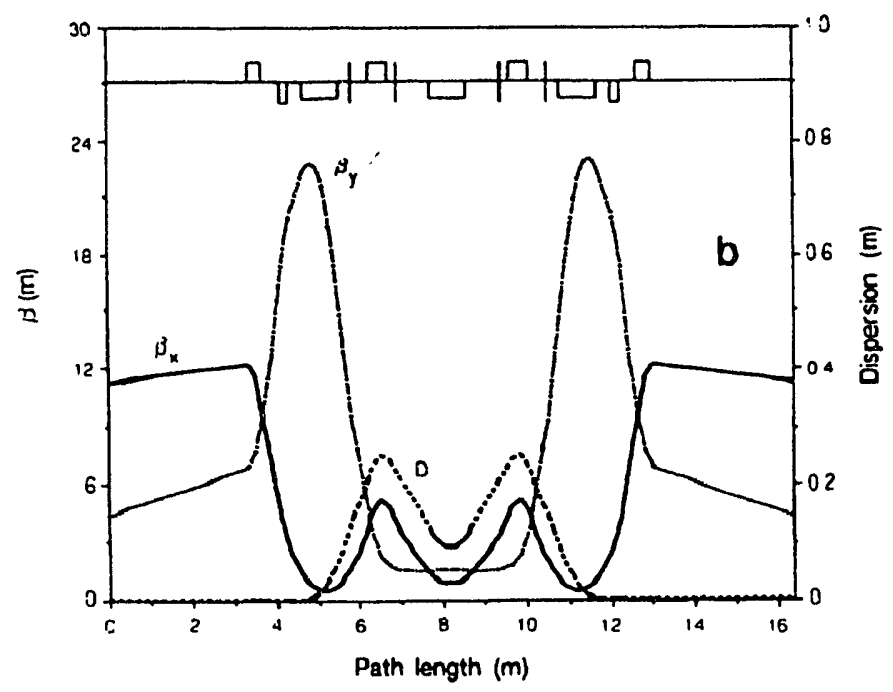

Figure 1.

(a) One superperiod of the ALS triple-bend achromat lattice.

(b) Horizontal and vertical $B$ functions and dispersion for one supesperiod of the storage ring lattice. 
Table 1

Main Parameters of the ALS Storage Ring

\begin{tabular}{|c|l|}
\hline Energy (nominal) & $1.5 \mathrm{GeV}$ \\
\hline (minimum) & $1.0 \mathrm{GeV}$ \\
\hline (maximum) & $1.9 \mathrm{GeV}$ \\
\hline Current (multibunch) & $400 \mathrm{~mA}$ \\
\hline (single bunch) & $8 \mathrm{~mA}$ \\
\hline Beam parameters at $1.5 \mathrm{GeV}$ & \\
\hline Natural emittance & $3.5 \mathrm{mn}$ \\
\hline Bunch length & $3.5 \mathrm{~mm}$ \\
\hline Energy spread & $6.5 \times 10^{-4}$ \\
\hline Radiation Loss/tum & $91.5 \mathrm{keV}$ \\
\hline
\end{tabular}

Table 2

Chronology of Storage Ring Commissioning

\begin{tabular}{|c|c|}
\hline Date & Event \\
\hline Jan. 13 & Personnel Safety System commissioned \\
\hline Jan. 15 & Beam through $2 / 3$ of circumference \\
\hline Jan. 19 & 1-1/3 turns established \\
\hline Feb. 2 & Discovered vertical scraper obscuring beam aperture \\
\hline Feb. 4 & $\begin{array}{l}\text { Found and fixed shorted coil on quadrupole magnet, } \\
\text { immediately achieving } 60 \text { turns with NO steering }\end{array}$ \\
\hline Feb. 8 & $\begin{array}{l}\text { DCCT commissioned, observed } 1.5 \mathrm{~mA} \text { injected } \\
\text { beam }\end{array}$ \\
\hline \multicolumn{2}{|c|}{$\begin{array}{l}\text { Main problems during this period: booster dipole power supply } \\
\text { failure; continuing failure of SR quad power supplies due to low } \\
\text { flow (all flow restrictors eventually removed); fast kicker magnet } \\
\text { failures; SR bend magnet power supply failures (two); BPM } \\
\text { system fault, requiring replacement of } 200 \text { PLA's. }\end{array}$} \\
\hline Mar. 16 & $\begin{array}{l}\text { RF system commissioned, } 6 \mathrm{~mA} \text { accumulated in } 4 \\
\text { bunches }\end{array}$ \\
\hline Mar. 24 & $\begin{array}{l}65 \mathrm{~mA} \text { accumulated in } 240 \text { bunches (DOE Project } \\
\text { Plan Technical Baseline) }\end{array}$ \\
\hline Apr. 9 & $\begin{array}{l}407 \mathrm{~mA} \text { in multibunch mode (c.f. } 400 \mathrm{~mA} \text { project } \\
\text { goal) }\end{array}$ \\
\hline Apr. 15 & $27 \mathrm{~mA}$ in a single bunch (c.f. $8 \mathrm{~mA}$ project goal) \\
\hline Apr. 30 & Beam lifetime 92 minutes (from $100 \mathrm{~mA}$ to $37 \mathrm{~mA}$ ) \\
\hline
\end{tabular}

\section{CHRONOLOGY OF STORAGE RING COMMISSIONING}

Three significant dates paced the progress of commissioning the storage ring: January 13, when the personnel protection system was brought into operation, defining the time when we could first bring beam into the storage ring; March 16, when the storage ring RF system was fully activated; and April 30, when commissioning was suspended in order to install IDs and beamlines. Major events and achievements during these periods are shown in Table 2.

\section{PERFORMANCE, LIMITATIONS, AND MISCELLAINEOUS OBSERVATIONS}

The primary performance indicators for the ALS are stored beam current (in single-bunch and multibunch modes), emittance, and beam lifetime. By the end of the commissioning period $460 \mathrm{~mA}$ had been accumulated in a filling pattern where 240 consecutive bunches, out of 328 , were filled there did not appear to be any fundamental limit at this current. In single-bunch mode, a current of $27 \mathrm{~mA}$ was achieved, apparently limited by a large coherent vertical oscillation. No optimization was altempted in this mode (by tuning the higher-order modes in the RF cavities, for example), since this current is already far in excess of the nominal single-bunch current of $8 \mathrm{~mA}$.

As described below, we were not instrumented to directly observe the beam emittance. However, those lattice parameters that determine the natural emittance were measured, and found to be consistent with the model. For some time at the beginning of the run, we were unwittingly operating with QFA set about 5\% low, leading to a natural emittance about a factor of two greater than nominal. This is the so-called "relaxed lattice" described in reference 2 . In the vertical plane the betatron coupling was measured to be much smaller than expected (a few tenths of $1 \%$ ); however, we observed vertical dispersion of around $2 \mathrm{~cm}$, which will lead to a vertical emittance between $1 \%$ and $10 \%$ of the natural horizontal emittance. This analysis is continuing.

Fri $3 / 19 / 93 \quad 8: 54: 43$

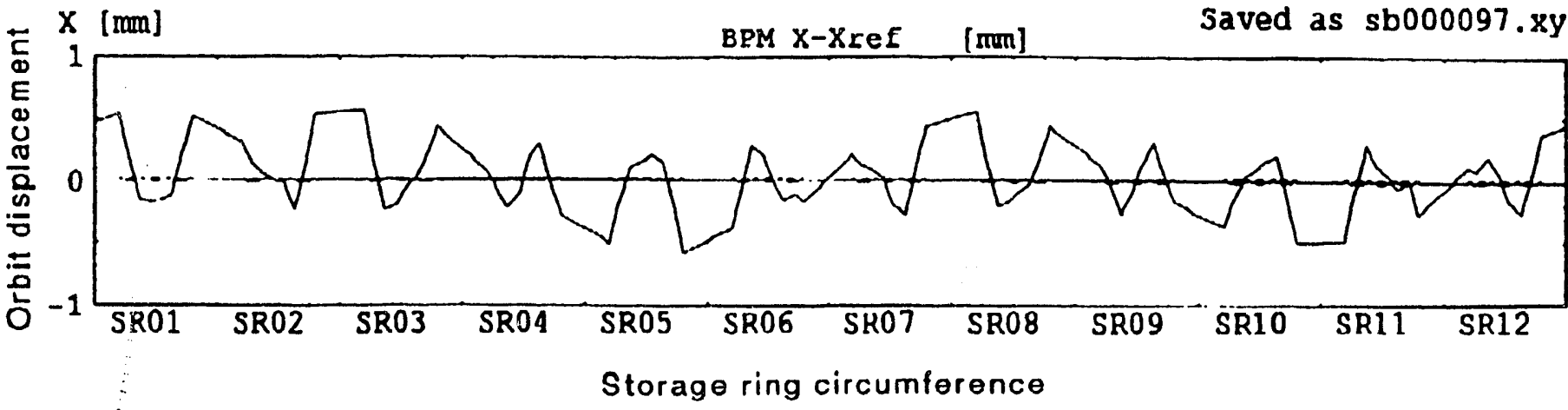

Figure 2. Change in horizontal closed orbit resulting from one corrector. Arrow marks the position of the corrector. 
Beam lifetime is consistent with elastic gas scattering lifetime of 30 hours at 1 ntorr, with an aperture of $18 \mathrm{~mm}$. Note that the vacuum pressure was typically 10 ntorr at current levels of $30 \mathrm{~mA}$, the ring was not baked, the powerful titanium sublimation pumps were not commissioned, and beam lifetime was improving at a rate of 2 to 3 minutes per day due to beam conditioning! No differences were observedbetween high currents in a few bunches and the same current in 240 bunches, indicating that the Touschek effect is not significant at lifetimes of 2 to 3 hours. No effects on lifetime due to ion trapping were observed: there were no significant differences in lifetime for different filling patterns, and there were no sudden losses of current after the beam was stored.

Accumulation rate is a secondary performance indicator. The injection efficiency was optimized at $65 \%$ (1.5 mA per shot in the storage ring from a circulating current of $6 \mathrm{~mA}$ in the booster). We expect to improve on the efficiency after the shutdown, with a better corrected horizontal orbit. Also, the injection rate will increase with improved booster current (to $15 \mathrm{~mA}$ ), expected as a result of implementing the beam-loading-compensation scheme in the linac.

\section{INSTRUMENTATION}

The full complement of storage ring instrumentation was not available during this commissioning period. In particular, the synchrotron light monitor, transverse kickers, and remotely adjustable scrapers were not installed, somewhat limiting the scope of measurements that could be made. These capabilities will be added during the current shutdown.

What were operational were th 96 beam position monitors (BPMs), each capable of providing turn-by-turn intensity and position signals, or more accurate time-averaged data, and three sets of higher fidelity button monitors (the LEP buttons), used with spectrum analyzers for fractional longitudinal and transverse tune measurements. The data from the BPMs could be analyzed on line with the ALS control system, or stored for further off-line analysis. Example are shown below.

\section{Vi. MEASUREMENT TECHNIQUES AND RESULTS}

Between March 16 and April 30, we accumulated a large amount of data. Some of this data, particularly that used to compare the real machine to our model, was analyzed in real time. However, a significant amount of analysis remains to be done, especially of data on instabilities and nonlinear dynamics. In this section we outline the scope of the studies undertaken, and present a summary of the results of our analyses to date.

\section{A. Closed-Orbit Distortions}

Closed orbits were measured by taking BPM data in the more accurate averaging mode. Absolute measurements of the orbits were used in conjunction with a local-bump, closedorbit-correction algorithm to successfully adjust the vertical orbit. An uncorrected orbit with peak-to-peak variations of 12 $\mathrm{mm}$ was reduced to one with an RMS displacement of less than $0.5 \mathrm{~mm}$ (see also reference 3). This same technique was not as successful in the horizontal plane for reasons not yet understood. By taking difference orbits it was possible to construct the full sensitivity matrix for the storage ring, i.e., the effect of each corrector magnet on the beam position all around the machine. An example of one such difference orbit is shown in Figure 2. By analyzing the data from homologous magnets in each sector we could quickly establish the excellent periodicity of the lattice $(<10 \%$ variation, corresponding to the accuracy of the measurement).

The same technique was also applied to empirically construct localized distortions across a straight section in order (eventually) to steer ID radiation down user beamlines. "Leakage" from the local bump was measured at less than $10 \%$, again limited by the accuracy of the measurement. By taking difference orbits as a function of RF frequency, it was possible to measure the dispersion function. Figure 3 shows a typical result. The first analysis of such data suggested that the QFA family of magnets were miscalibrated by a factor of $5 \%$. The power supply calibration was checked and indeed found to be in error by 5.5\%! Dispersion was also "driven" by frequency modulating the RF, which permitted real-time tuning to minimize dispersion in the straight sections.

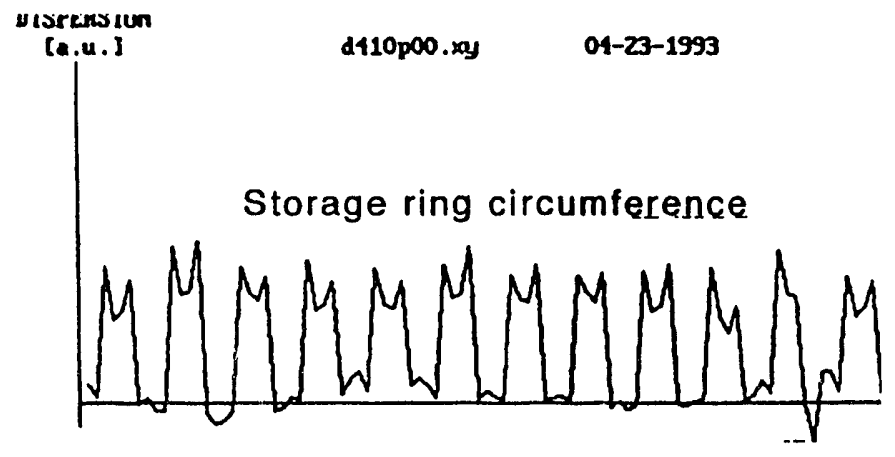

Figure 3. Dispersion function created by taking difference orbits at different RF frequencies.

\section{B. Tune Measurements}

Betatron tunes could only be measured because of the residual oscillations arising from the imbalance of the injection bump magnets (amounting to about $1 \mathrm{~mm}$, in the horizontal plane only), or from the small oscillations set up by transverse instabilities. An attempt was made to drive oscillations through powering the LEP buttons with an amplified tracking generator signal. This was partially successful in the horizontal plane (where good data already exists!), but not successful in the vertical plane. Tunes were measured through either the spectrum analyzers, or more often by Fourier analysis of the turn-by-turn data from the 96 BPMs. Despite this inconvenience, a huge amotint of data was taken. Variation of tunes as a function of quadrupole and bend magnet settings has been used to calibrate the storage ring model, to measure 
transverse coupling, and to measure and correct the chromaticity. After chromatic correction the data is good enough to show the nonlinear chromaticity, which agrees well with our model predictions. A typical set of data, showing the variation of betatron tunes through a coupling resonance, is shown in Figure 4, the data indicate an emittance coupling ratio of less than $1 \%$. Also, nonlinear tune shifts were measured by exciting different betatron oscillation amplitudes at injection.

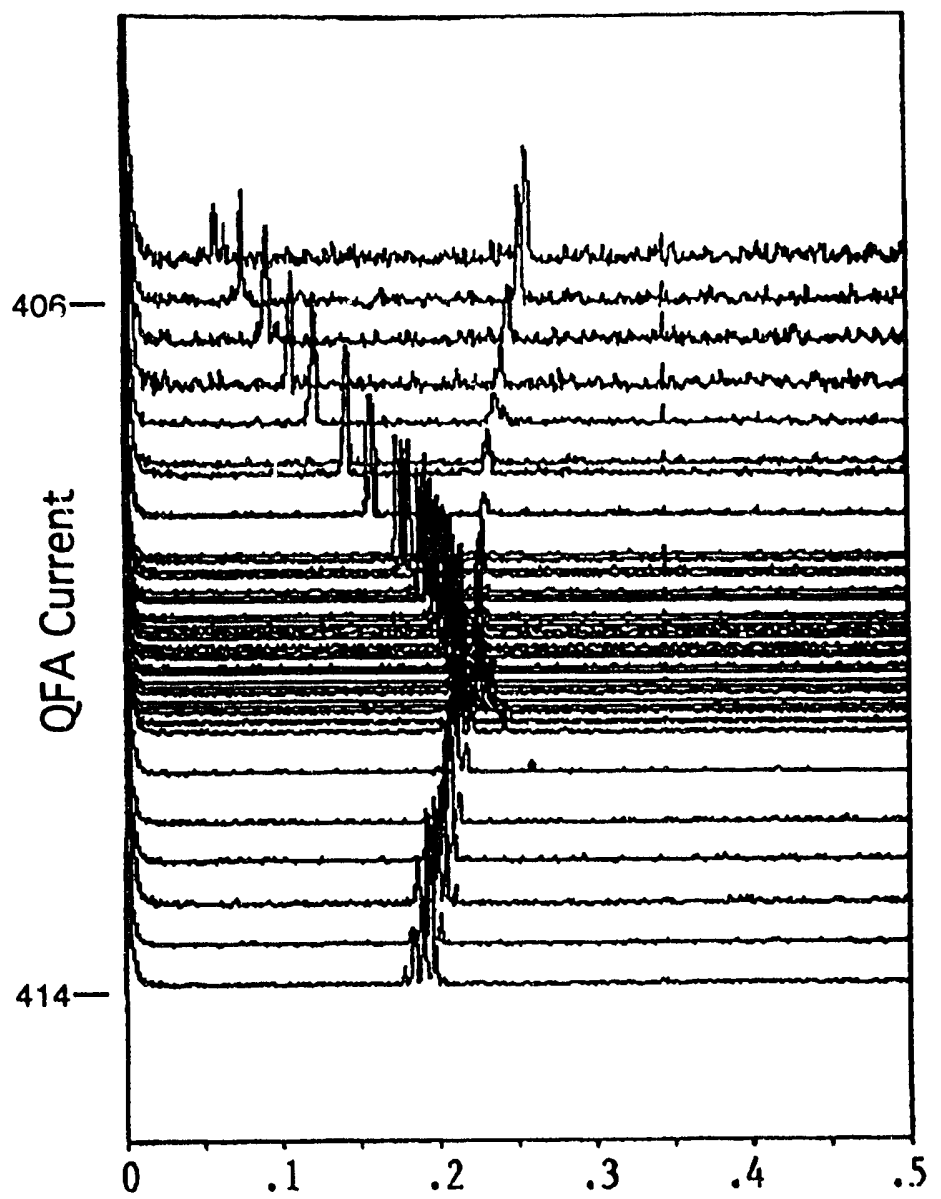

Fractional Tune

Figure 4. Tune scans as a function of QFA current, constructed from Fourier transforms of turn-by-turn orbit data.

Synchrotron tunes were always easy to measure, even at low beam current. Their variation as a function of RF voltage (from a nominal value of $10 \mathrm{kHz}$, where $\mathrm{fs}=0.007$ ) was used to estimate RF cavity voltage and power.

\section{Instability studies}

Phase oscillations arising from multibunch instabilities, limiting at about $10^{\circ}$, were observed in a filling pattern where 82 equidistant bunches were filled; however, this instability did not appear to limit the amount of current that could be accumulated. The spectrum of synchrotron side-bands around the orbit harmonics were in good correspondence to the aliased longitudinal higher-order modes in the accelerating cavities (see reference 4). In single-bunch mode, the longitudinal damping system, being developed jointly with SLAC, was tested and successfully damped a deliberately driven oscillation to less than $1^{\circ}$. Measurements of the width of the synchrotron side-bands gave an upper limit to the bunch length of $8 \mathrm{~mm}$, or $27 \mathrm{ps}$ FWHM. Single-bunch currents up to $27 \mathrm{~mA}$ were accumulated.

Measurements were also taken on the effects of the newly injected beam (with large betatron oscillations), on the already stored beam, to compare with models recently developed in collaboration with SLAC . . . analysis is in progress.

\section{SUMMARY}

Commissioning the ALS storage ring was undertaken in two periods. The first, with no RF acceleration available, was extremely useful in debugging the instrumentation and controls, making the hardware reliable, checking the efficacy of the environmental shielding, and making preliminary measurements on injection and the computer model. The second phase lasted less than seven weeks, in which time all the project goals at $1.5 \mathrm{GeV}$ were met, and a huge amount of data was taken towards understanding the parameters of the storage ring. During the months through July 1993, the IDs and beamlines will be installed in preparation for establishing the ALS as a full user facility.

\section{ACKNOWLEDGMENTS}

The work presented here was carried out by the ALS Accelerator Physics Section and Operations Section. The sheer volume of work could not have been accomplished without the help of our visitors, particularly Peter Kuske and Bettina Kuske from BESSY, and from members of the LBL Center for Beam Physics. Instrumentation and controls naturally played a major role in the success of the commissioning activities and it is a pleasure to acknowledge the response to requests for "urgent" changes provided by Jim Hinkson and Chris Timossi.

This work was supported by the director, Office of Energy Research, Office of Basic Ene:gy Sciences, Material Sciences Division of the U.S. Department of Energy, under Contract No. DE-AC03-76SF00098.

\section{REFERENCES}

[1] See for example A. Ropert, "The Status of the ESRF," in Proceedings of the Third European Particle Accelerator Conference, Berlin '92, pp. 35-39.

[2] Lawrence Berkeley Laboratory PUB-5172, "1-2 GeV Synchrotron Radiation Source, Conceptual Design Report," 1986.

[3] L. Schachinger and V. Paxson, "A Software System for Modeling and Controlling Accelerator Physics Parameters at the ALS," these proceedings.

[4] J. Qyrd, J. Corlett, "Coupled-bunch Stability at the ALS," these proceedings. 

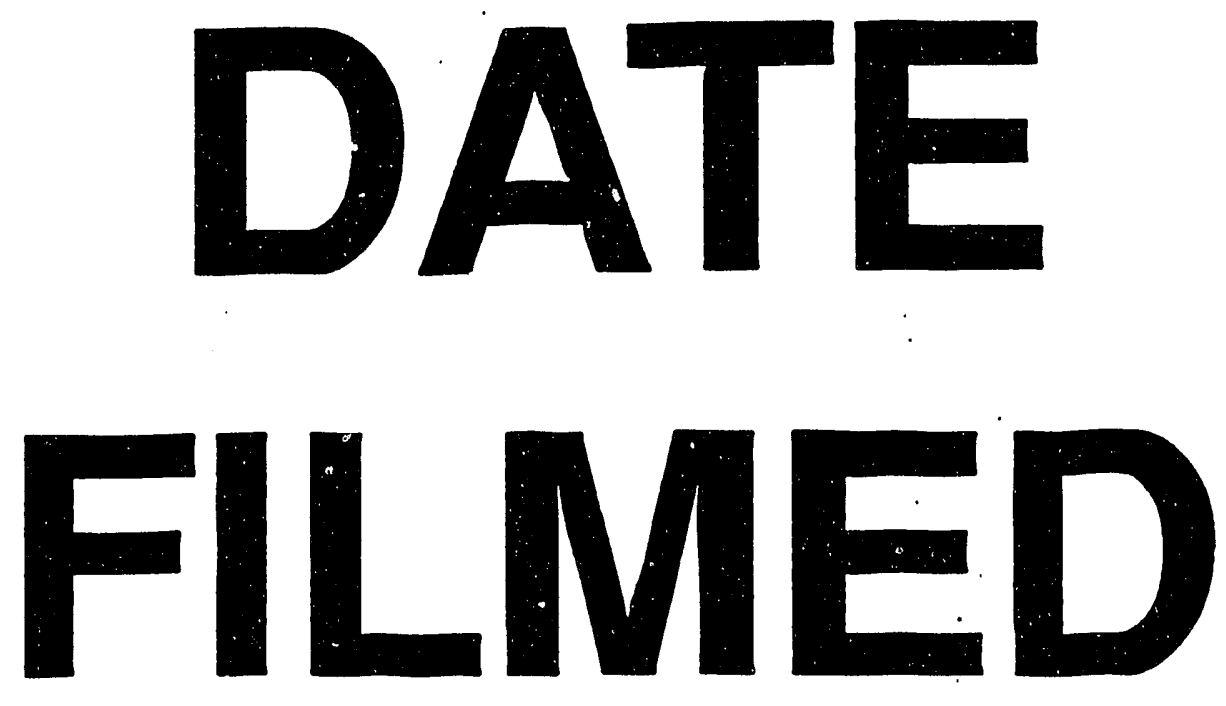

$10 / 12 / 93$
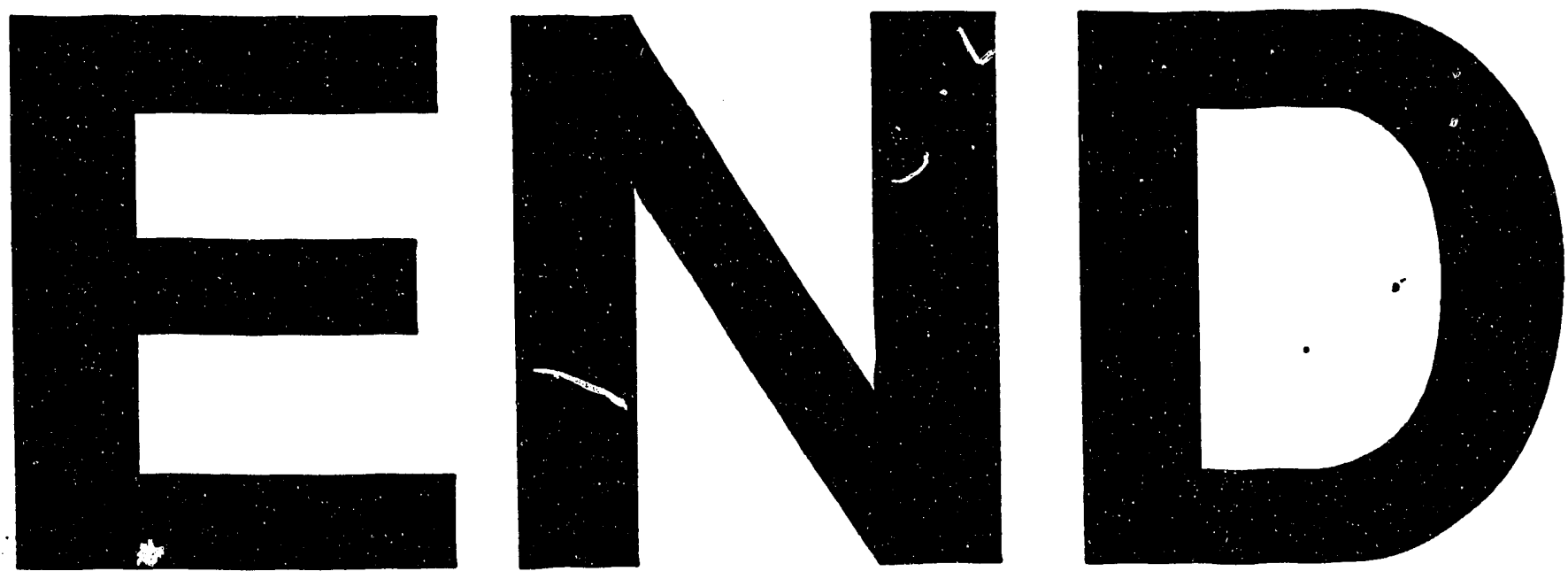
